Russian Academy of Science, Siberian Branch

BUDKER INSTITUTE OF NUCLEAR PHYSICS

A.A. Ivanov, P.P. Deichuli, A. Kreter, V.V. Maximov, A.A. Podyminogin, B. Schweer,

I.V. Shikhovtsev, N.V. Stupishin,

R. Uhlemann, D.V. Usoltsev

CHARACTERIZATION OF ION SPECIES MIX

OF THE TEXTOR DIAGNOSTIC

HYDROGEN BEAM INJECTOR

WITH AN RF AND ARC-DISCHARGE

PLASMA BOX

Budker INP 2002-41

Novosibirsk

2002 


\title{
Characterization of ion species mix of the TEXTOR diagnostic hydrogen beam injector with an RF and arc-discharge plasma box
}

A.A.Ivanov, P.P.Deichuli, A.Kreter* , V.V.Maximov,

A.A.Podyminogin, B.Schweer*, I.V.Shikhovtsev, N.V.Stupishin,

R.Uhlemann*, D.V.Usoltsev

Budker Institute of Nuclear Physics

630090, Novosibirsk, SB RAS

*Institut fuer Plasmaphysik, Forschungszentrum Juelich GmbH,

EURATOM Association, Trilateral Euregio Cluster, 52425 Juelich, Germany

\begin{abstract}
The ion species fractions of the diagnostic hydrogen beam of the tokamak TEXTOR were determined by means of the $\mathrm{H}_{\alpha}$ Doppler shift spectroscopy and alternatively by a magnetic massspectrometer. Both methods provided similar quantitative assessment of the ion species mix. The measurements were done for a fixed beam energy of $50 \mathrm{keV}$ and a variable beam current of up to $2.5 \mathrm{~A}$. The ion source at the test stand was operated with two alternative types of plasma boxes, RF and arc discharge based, which provided the same ion current density and total extracted ion current. According to the measurements, the density component of the beam particles with the full energy is about $50 \%$ for the RF plasma box and more than $75 \%$ for the arc discharge plasma box.
\end{abstract}

(C) Budker Institute of Nuclear Physics SB RAS 


\section{Introduction}

The hydrogen diagnostic beams are widely exploited in magnetic fusion devices [1-4], providing measurements of local parameters of the plasma and magnetic field. In particular, the diagnostic neutral beam injector of the tokamak TEXTOR is used for the measurements of the ion temperature and impurity profiles via active charge-exchange recombination spectroscopy (CXRS) [4]. As well as the beam angular divergence and current density, the beam species mix is critically important for this diagnostic. Neutral hydrogen beams usually contain atoms with three different energies: $\mathrm{H}(E), \mathrm{H}(E / 2)$ and $\mathrm{H}(E / 3)$, originating from atomic and molecular ions extracted from the ion source with the same kinetic energy $E$. The full energy species fraction influences the CXRS diagnostic signals in several ways. Firstly, the rate coefficient of the $\mathrm{H}^{0}+\mathrm{C}^{6+}$ charge-exchange reaction, which is routinely used for the ion temperature measurements, has a quite sharp maximum near $50 \mathrm{keV}$ energy of hydrogen atoms, corresponding to the full energy component of our beam. Secondly, the penetration depth of the lower energy species into the dense plasma core is significantly smaller. Therefore, a higher full energy species fraction in a hydrogen beam enables to provide higher signals in the detection system.

The ion species distribution can be deduced from the $\mathrm{H}_{\alpha}$ Doppler shift spectroscopy $[5,6]$. This spectroscopic technique has first been applied to neutral beam injectors for plasma heating [5,7] and later on also to diagnostic neutral beams $[8,9]$. In the measurements we followed to the standardized procedure described in [7] to evaluate the beam species mix by taking into account the ratios of the integrated Doppler-shifted light peaks of the full, half and third energy components and multiply them by factors, which contain the excitation cross section and beam fractions. It was assumed, that the observed intensities of the light emitted by the beam species are governed by the Corona model, i.e. the radiative decay rates balance the collisional excitation of the corresponding level. This is legitimate for low background gas densities, at which the collisional deexcitation can be neglected. In accordance with the model used [7], the ratio of densities of the beam species was measured with the spectroscopic method.

Beside the spectroscopic method, the beam species distribution has also been measured by means of the magnetic mass-spectrometry. For this purpose, a particle energy analyzer was installed behind the beam dump on the beam axis. This method provided the direct measurements of the current distribution of beam 
particles with different energies, instead of the species densities as for the spectroscopic measurements, and can be taken as the referential one.

The measurements have been performed for the diagnostic beam source equipped with different plasma boxes, RF and arc discharge based ones, to compare their beam species composition.

\section{Ion source of the diagnostic neutral beam injector}

Version with RF-discharge plasma box

The RF-discharge ion source is used in the diagnostic injector at TEXTOR. In the injector ion source (see figure 1) an ion current of $\sim 1.8 \mathrm{~A}$ is routinely extracted and accelerated by a four-grid ion optical system to an energy of $50 \mathrm{keV}$. The grids were made of molybdenum with 163 holes of $4 \mathrm{~mm}$ diameter arranged into a hexagonal pattern. In order to provide beam focusing, the grids were formed to spherical segments with a radius corresponding to the desired focal length.

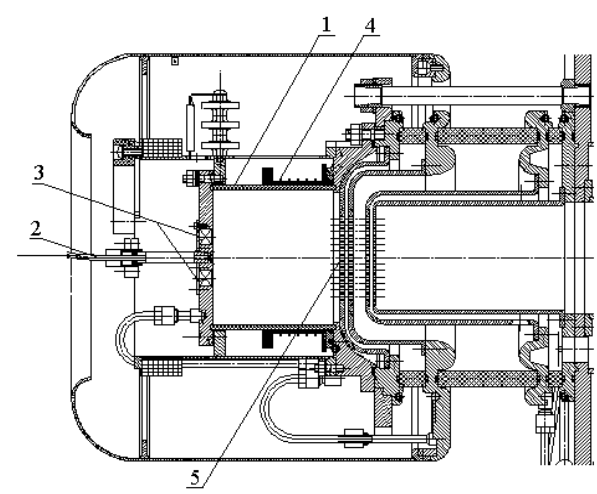

Figure 1. Ion source with RF plasma box: 1 - ceramic discharge chamber; 2 - gas inlet; 3 - permanent magnets; 4 - RF coil; 5 - grids.

Originally, the ion source has been designed to operate with the RF plasma box, which has a cylindrical discharge chamber made of alumina ceramic with a wall of $4 \mathrm{~mm}$ thickness. The rear copper flange and the spacer between the ceramic tube and the plasma grid are water-cooled. At the rear flange, there are a gas inlet and a trigger device which is used to initiate the discharge. The discharge is excited by a 6-turns coil installed at the ceramic chamber. The coil is connected to a $\quad 4.65 \mathrm{MHz}$ amplifier through an insulating oil transformer. The experimentally verified data of the diagnostic beam are given in table 1 . This type of the ion source is described in detail in $[3,4]$. 
Table 1. Ion source data of the diagnostic beam injector at TEXTOR

\begin{tabular}{l|l}
\hline Beam energy & $20-52 \mathrm{keV}$ \\
\hline Beam current & up to $2.3 \mathrm{~A}$ \\
\hline Pulse duration (with $500 \mathrm{~Hz}$ modulation) & max. $10 \mathrm{~s}$ \\
\hline Divergence & $\sim 0.6^{0}$ \\
\hline Focal length & $4 \mathrm{~m}$ \\
\hline Grid transparency & $57 \%$ \\
\hline Extraction area & $6.8 \mathrm{~cm}$ in diameter \\
\hline
\end{tabular}

Version with arc-discharge plasma box

As it was mentioned in Introduction, an increase of the full energy component current is of prime concern for the TEXTOR diagnostic beam for accurate measurements of the CXRS signals. This is especially important for high density regimes of the tokamak operation, when the beam attenuation becomes very significant. Therefore, it was proposed to test an arc-discharge version of the plasma box for the diagnostic beam, which is potentially capable of providing a higher proton fraction because of a higher power density in the discharge. General view of the arc-discharge plasma box is shown in figure 2. This version of the plasma box was fully compatible with the ion-optical system of the diagnostic beam.

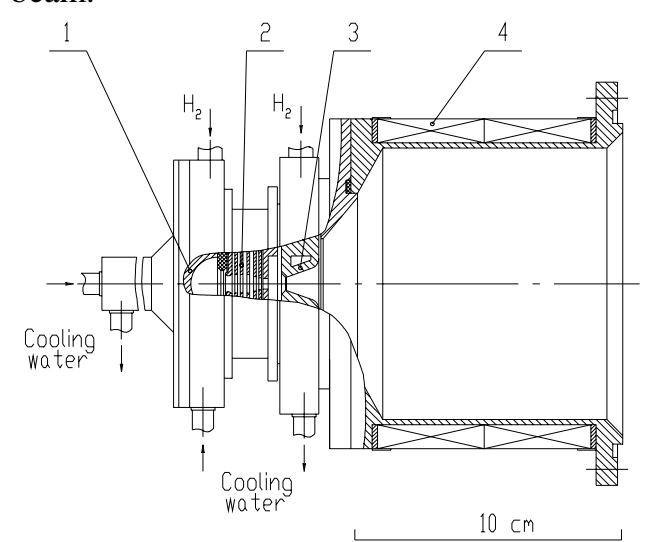

Figure 2. Schematic view of arc discharge plasma box: 1 - arc plasma source, 2 - cathode, 3 anode, 4 - permanent magnets.

The plasma stream was produced by the arc discharge plasma source (see figure 3) and subsequently expanded into a region with peripheral magnetic field, which was produced by a picket fence magnet (figure 2). As a result of the ion reflection by the magnetic field, a density profile with the required uniformity was formed at the plane of the plasma grid. Magnetic field in the expander periphery 
was produced by an array of $16 \mathrm{Nd}-\mathrm{Fe}-\mathrm{B}$ permanent magnets of $8 \mathrm{~cm}$ length and $1 \mathrm{~cm}$ width each. Magnetic field strength at the inner wall of the expander was 0.2 $\mathrm{T}$ and fallen down radially to a level of less than $0.01 \mathrm{~T}$ at $2 \mathrm{~cm}$ distance from the inner wall.

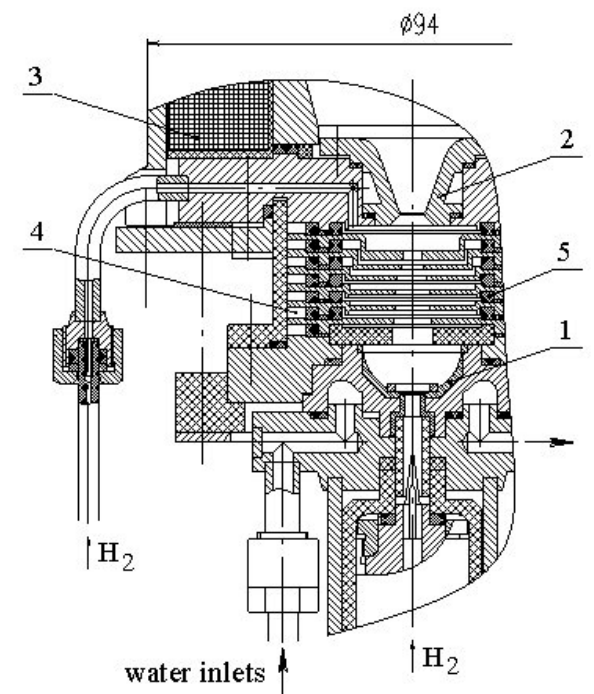

Figure 3. Cutaway drawing of the arc-discharge plasma source. 1 - cathode, 2 anode, 3 - magnetic insulation coil, 4 - cooling water channels, 5 - insulating washer.

The plasma source had an augmented cooling of the electrodes and washers to ensure the stationary operation. The copper cathode of the source had a spherical cavity of $1 \mathrm{~cm}$ in diameter. The cathode was cooled by water supplied thought a ring-shaped channel in the cathode body with a wall thickness of 2-3 $\mathrm{mm}$. The anode copper insert had an inner water cooling loop. The washers were also cooled from their edges by a water flow. The gas was supplied through a hole in a special triggering electrode located at the center of the cathode. The discharge was initiated by applying a high voltage triggering pulse between the cathode and the trigger electrode (figure 3).

According to a movable gridded analyzer measurements, the radial profile of the plasma density at the plane of plasma grid had a non-uniformity of $\pm 4 \%$ inside a circle with radius of $4.5 \mathrm{~cm}$. The arc plasma source was operated in up to $2 \mathrm{~s}$ pulses providing an ion current density of the emitter in the range of $\sim 130 \mathrm{~mA} / \mathrm{cm}^{2}$ for a discharge current of $300 \mathrm{~A}$. 


\section{Experimental setup for magnetic and spectroscopic measurements}

\section{Magnetic mass-spectrometer}

As it has already been pointed out, an essential parameter of the diagnostic beam are the density (or current) fractions $\mathrm{H}^{0}(E), \mathrm{H}^{0}(E / 2)$ and $\mathrm{H}^{0}(E / 3)$, which appear after the beam passed through the neutralizer and a deflection magnet in the beam line. These fractions originate from $\mathrm{H}^{+}, \mathrm{H}_{2}{ }^{+}$and $\mathrm{H}_{3}{ }^{+}$ions of the extracted beam. Their relative currents were measured at the test stand at the Budker Institute in Novosibirsk by using a magnetic mass spectrometer located on beam axis $5 \mathrm{~m}$ downstream from the source. A schematic diagram of the mass spectrometer layout is given in figure 4 . The beam entered the analyzer through a $4 \times 2.5 \mathrm{~mm}$ slit and passed the stripping cell filled with helium. To provide an equilibrium target for the charged and neutral beam components, the gas pressure in the stripping cell was sustained to be high enough during the measurements. For that purpose, a fast pulsed valve served to provide a localized gas cloud inside the stripping chamber.

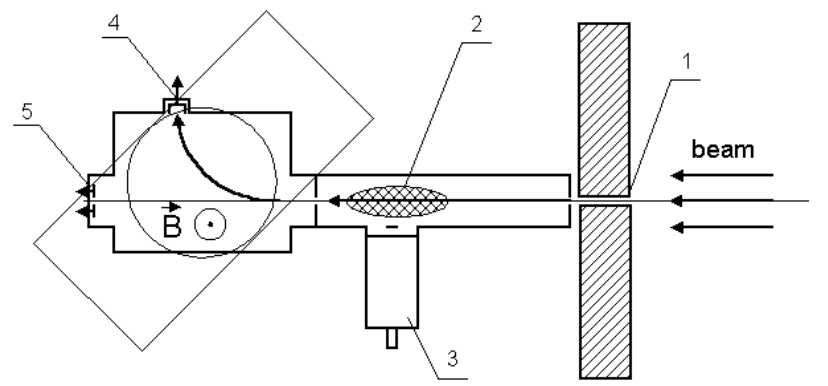

Figure 4. Magnetic mass spectrometer. 1 - entrance slit; 2 - pulsed He target; 3 gas inlet valve; 4, 5 - Faraday cups.

After passing the pulsed stripping target, the beam entered a magnet deflecting the particles by $90^{\circ}$. The ion current of each species was measured by a Faraday cup. To measure the different energy components the magnetic field was varied accordingly.

\section{Spectroscopic measurement}

Two alternative optical systems were simultaneously used for the spectroscopic measurements of the species mix. The first one (figure 5) is routinely used on the test stand for the optical measurements. The beam was viewed under an angle of $30^{\circ}$ by an optical system consisting of a lens, a prism and a mirror, which focused the light on the entrance slit of a grating monochromator. The spectrally 
resolved light reflected from the grating was focused on the entrance of a dissector after passing through an objective with a $50 \mathrm{~mm}$ focal length. The image was scanned over the $0.1 \mathrm{~mm}$ slit of the dissector and transmitted to an ADC together with the base generator signal. With a 1200 grooves/mm grating, a focal length of $600 \mathrm{~mm}$ and $0.2 \mathrm{~mm}$ width of the monochromator entrance slit the resulting spectral resolution of the system was about $3 \AA$. Taking into account an image magnification factor of the optical system of 0.5 , one can estimate the dimensions of the volume the light was collected from as a region $0.4 \mathrm{~mm}$ along the beam and $36 \mathrm{~mm}$ in the transverse direction.

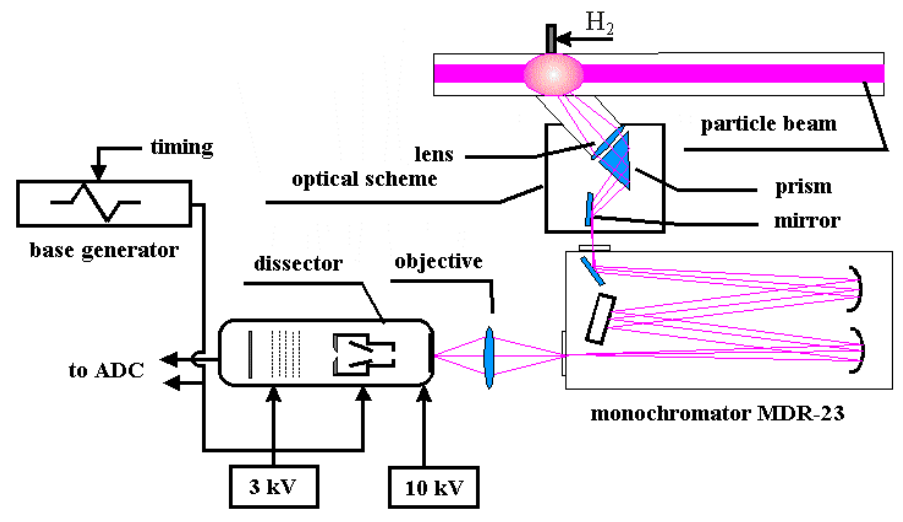

Figure 5. Schematic layout of spectrometer and optical setup.

For the series of experiments presented in this article the test stand was additionally equipped with an optical system provided by the German co-authors. Here, the beam emission was observed under an angle of $40^{\circ}$ by an optical fiber, which transported the collected light to a compact grating spectrometer equipped with a CCD line array detector.

In order to provide high enough intensity to the both observation systems in the case of a small beam duration or/and current, a $150 \mathrm{~mm}$ diameter quartz tube was installed inside of the vacuum chamber in which a localized gas cloud could be produced by using a pulsed gas valve. This tube was mounted on a vacuum tight displacement mechanism enabling to move it into the position co-axially to the beam or move it completely out. At both ends of the tube there were $55 \mathrm{~mm}$ diameter apertures for the beam collimating. Unless otherwise stated, the data presented below were taken with this tube installed on the beam axis and the gas flowed into it. When the beam provided enough light for the observation system, we were able to compare the results obtained with and without the gas puff into the tube. Apart from the overall increase of the light intensity, the measurement with 
the gas flow provided a beam composition similar to that obtained without the additional gas.

\section{Measured ion species mix}

$\underline{\text { RF plasma box data }}$

Typical signal measured by the magnetic mass spectrometer for the ion source with RF plasma box is presented in figure 6. In this case, the beam current was $1.7 \mathrm{~A}$, and hydrogen gas flow rate into plasma box was 1 Torr l/s. For these parameters, the ion species mix in the extracted beam current measured by magnetic mass-spectrometer was $\mathrm{H}^{+}-66 \%, \mathrm{H}_{2}{ }^{+}-26.5 \%, \mathrm{H}_{3}{ }^{+}-5 \%$ and water $\sim 1.5 \%$.

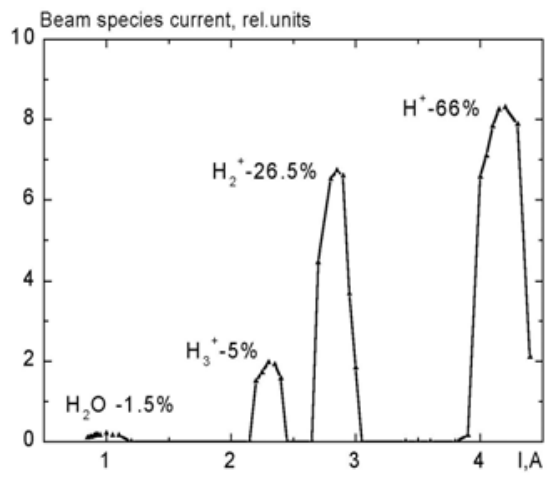

Figure 6. Signal measured by magnetic mass spectrometer vs magnet coil current.

It was observed that the beam composition varied either with the absorbed RF power and the gas flow rate. For the studied range, the general tendency was that the full energy species increased with both higher RF power and gas puffing. Note that the beam current was increased with an increase of the both parameters. Accordingly, the maximal full energy species current fraction of $\sim 70 \%$ was achieved for the beam current of $2-2.3 \mathrm{~A}$, which was the maximum beam current obtained in these experiments.

For the beam formation with the arc-discharge and RF plasma emitters we used a grid system, which is identical to that of the TEXTOR diagnostic injector. The beam profile was monitored by an array of secondary emission detectors and miniature calorimeters. Typically, the beam was extracted during $100-200 \mathrm{~ms}$, whereas the spectroscopic data were collected during $80 \mathrm{~ms}$. Prior to the beam extraction, a pre-shot plasma discharge in the plasma box was initiated and sustained during $50 \mathrm{~ms}$ for the conditioning of the inner walls of the plasma box.

As an example of the data provided by the spectroscopic measurements, figure 7 shows several well-separated light peaks for the $50 \mathrm{keV}$ hydrogen beam extracted with the RF version of the plasma box. The observed shifts of the peaks 
from the unshifted $\mathrm{H}_{\alpha}$ light emitted by the background gas were in good agreement with the calculated values for the given beam energy and observation angle.

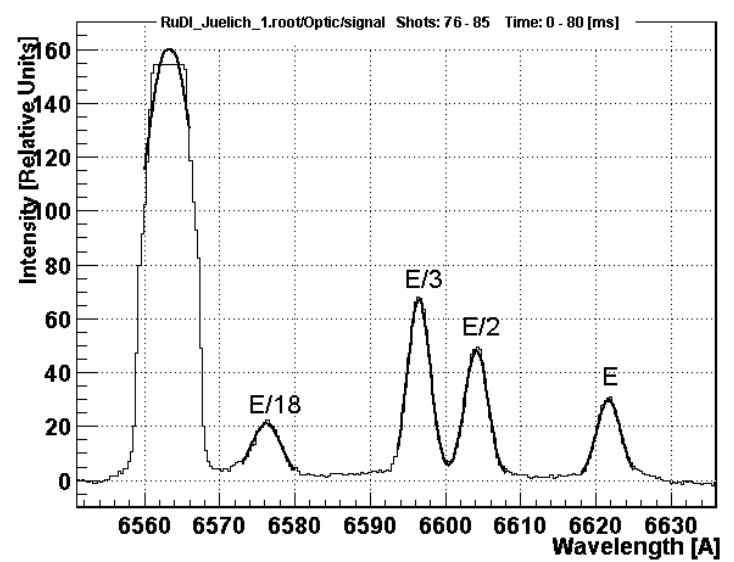

Figure 7. $\mathrm{H}_{\alpha}$ - light spectrum of $50 \mathrm{keV}, 1.75 \mathrm{~A}$ - hydrogen beam ("old” RF plasma box is employed). Proton species is $44 \%$ by density.

In this series of shots the RF-plasma box was employed, which has been operated at TEXTOR for about two years, so that its integrated beam-on time amounts to approximately $2 \cdot 10^{4} \mathrm{~s}$. During the operation of this ion source at TEXTOR it was observed, that the beam species mix slowly degraded, so that the full energy component substantially decreased. Initially, it was measured to be $\sim 50 \%$, instead of $\sim 40 \%$ obtained after a long operation of the source. This observation was proven by installing a RF plasma box with a new ceramic cylinder. Corresponding spectrum showing the different beam species ratios is shown on figure 8. We attributed the observed difference to a modification of the surface layer of the ceramic chamber walls. For a new, clear ceramic the recombination of atomic hydrogen at the surface is expected to be very small [10]. After a long operation of the source, it was noticed that the ceramic surface was eventually covered by a gray conductive layer. It indicated appearing of metallic aluminum on the surface, that may result in an increased recombination leading to the reduction of the proton fraction in the discharge.

Variation of the ion species mix with the extracted beam current and gas puffing rate was studied by using the optical systems with the dissector and the CCD camera. Both detection systems provided reasonably close results (see figure 9).

Figures 10, 11 show variation of the specie currents with the extracted beam current and gas puff rate. For comparison, in figure 10 the spectroscopic data on 
the beam specie densities are presented, but recalculated for the ion species current. Note quite good agreement of both methods used.

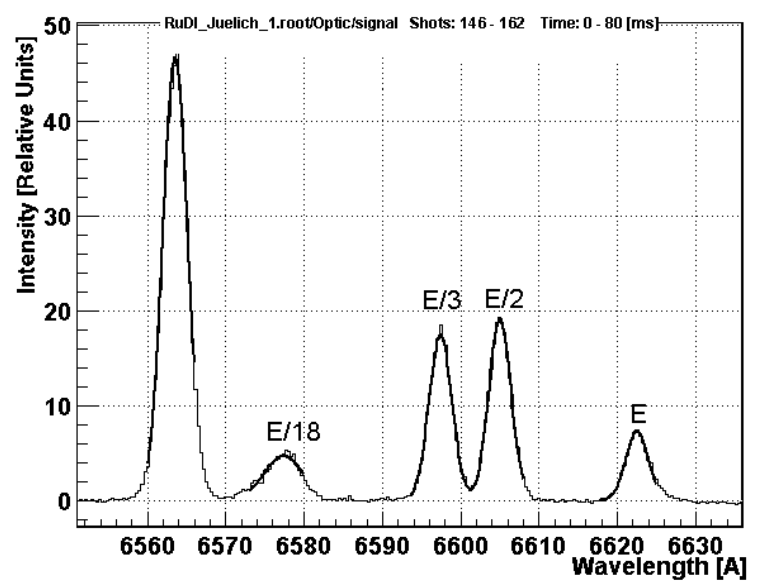

Figure 8. Doppler-shifted spectrum of the beam (new plasma box). proton species is $52 \%$ by density.

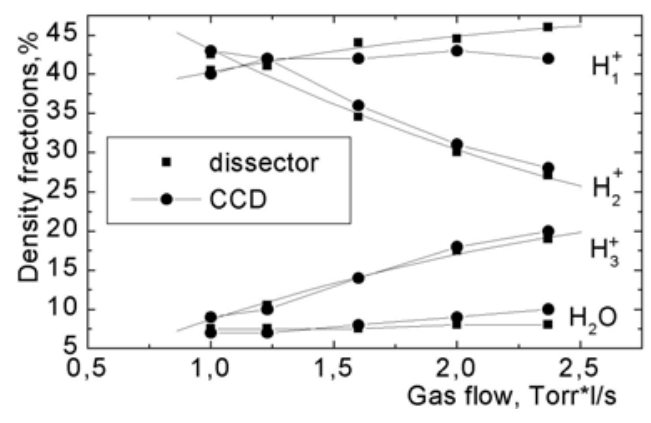

Figure 9. Beam species density vs gas flow rate for beam current of 1.8 A measured with dissector and CCD camera. 


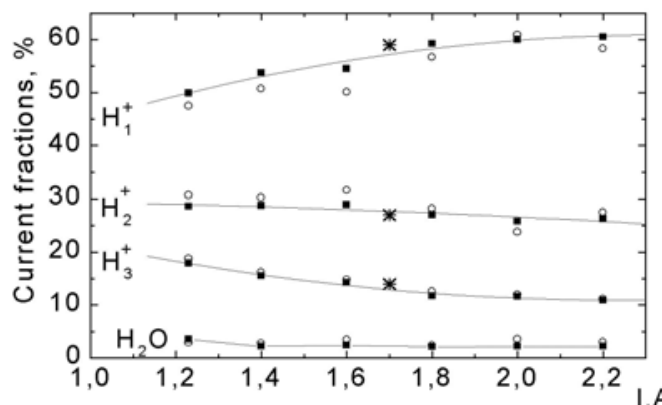

Figure 10. Beam species current vs extracted beam current. Gas flow rate in the source is 2 Torr $\cdot \mathrm{l} / \mathrm{s}$. Circles stand for the CCD data recalculated for the species current, star symbols stand for the mass spectrometer data.

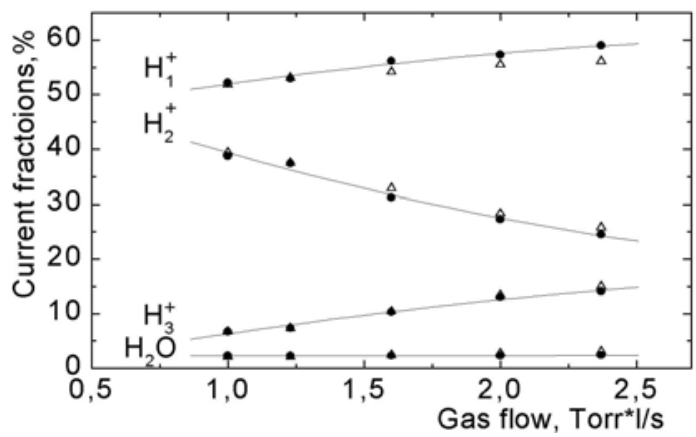

Figure 11. Beam species current vs gas flow rate for beam current of $1.8 \mathrm{~A}$. Triangulars stand for CCD data.

A special series of the experiments was performed to check whether the measured species ratios were sensitive to the gas pressure in the observation region. In these experiments we varied the amount of gas puffed into the glass tube. Some results are shown in figure 12 . This figure essentially represents the same data as in figure 10, but includes also the data taken with varying the gas density in the tube. Namely, points labeled $1-5$ were measured for the gas density varied by approximately 5 times in magnitude. Additionally, in separate experiments the bending magnet of the diagnostic beam injector was activated that caused changes in the beam species mix and in the beam emission spectra. At the same time, while the gas target in the neutralizer was thick enough providing the charged beam fractions were in equilibrium, one can re-calculate these data, so as again to obtain the species mix in the extracted beam [7]. These data are represented by the points 4,5 . Note that the gas density in the glass tube differed in these points by 5 times. 


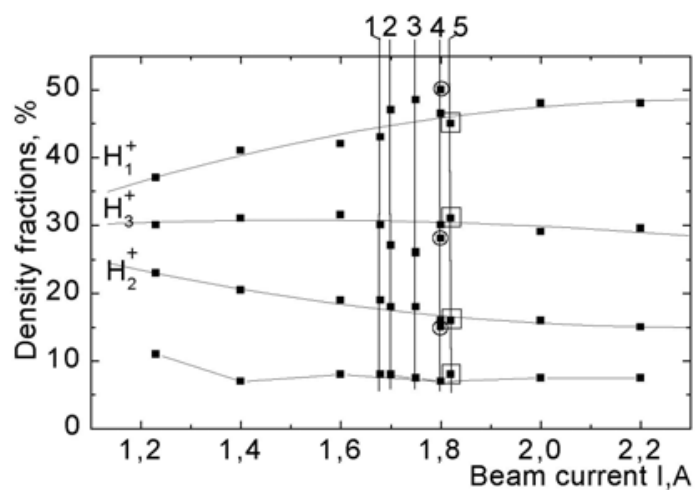

Figure 12. Beam species relative density. Gas flow rate in the source 2 Torr $1 / \mathrm{s}$.

Thus it was proven, that for the gas densities in the tube that were realized in the experiments, the deduced beam species did not vary significantly. The data shown in figure 12 also support applicability of the used model of the density species reconstruction to our experimental conditions. Indeed, the data obtained with the magnetic mass-spectrometer are reasonably close to the results of the optical measurements (indicated by stars in figure 12).

\section{Arc-discharge plasma box}

It was expected, that the plasma produced in an arc-discharge plasma box had a higher proton fraction. The reason for it is that the plasma density inside the ion source near the anode hole is quite high, of the order of $10^{15} \mathrm{~cm}^{-3}$ or higher. For such high density, the gas puffed into the anode region is almost completely ionized at the exit from the anode cavity. Accordingly, the molecular ion fractions decrease in the plasma very fast due to frequent collisions causing their dissociation. Subsequently, the plasma expands into the expansion volume towards the plasma grid. During this very rapid expansion the plasma density dramatically falls down, so that its value near the plasma grid amounts to about $10^{12} \mathrm{~cm}^{-3}$ (at an ion current density of $\sim 100 \mathrm{~mA} / \mathrm{cm}^{2}$ ). Therefore, the additional gas ionization in the expansion volumes is negligible and molecular ions do not appear. This general consideration was supported by the measurements with the arc-discharge plasma box. Note, that the current density and the total beam current were about the same for the RF- and the arc-discharge plasma boxes. A typical Dopplershifted spectrum of the beam light measured with the arc-discharge plasma box is shown in figure 13. 


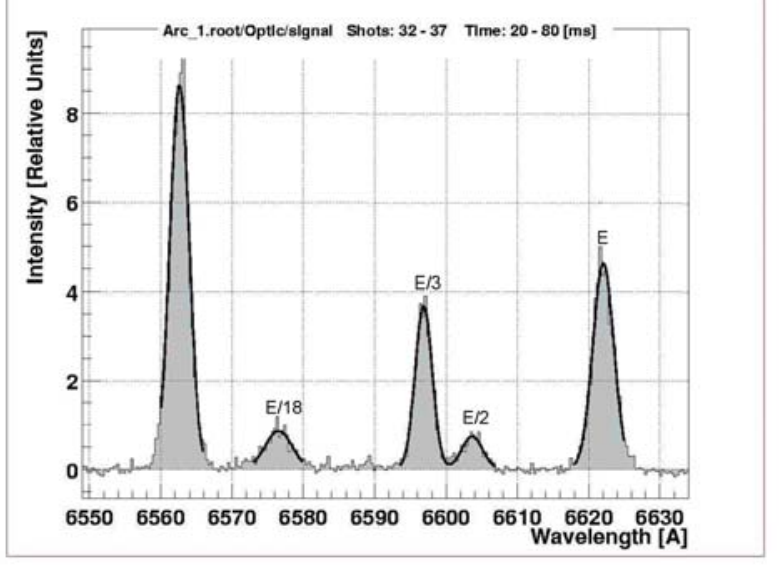

Figure 13. Spectrum of the $\mathrm{H}_{\alpha}$ lines emitted by a $50 \mathrm{keV}, 1.7$ A beam produced by the ion source with arc-discharge plasma box. Species density is $80 \%$ for $\mathrm{H}^{+}, 5 \%$ and $15 \%$ for $\mathrm{H}_{2}^{+}$and $\mathrm{H}_{3}{ }^{+}$, correspondingly.

As in the case of the RF version of the ion source, the beam composition measured with the magnetic mass-spectrometer was found in a reasonable agreement with the data presented in figure 13. Indeed, it provided the following species mix (not including the water, which was between $1-2 \%$ in this case): $\mathrm{H}^{+}$ $-87 \%, \mathrm{H}_{2}{ }^{+}-5 \%, \mathrm{H}_{3}{ }^{+}-8 \%$ (by current). In terms of the density in different species it corresponds to $\mathrm{H}^{+}-80.6 \%, \mathrm{H}_{2}{ }^{+}-6.6 \%, \mathrm{H}_{3}{ }^{+}-12.8 \%$, that reasonably agrees with the data given in figure 13 .

Variation of the ion species mix with the beam current is shown in figure 14. Here the data, which were taken with the Doppler spectroscopy, are presented by black box symbols. The other points represent the data of magnetic massspectrometer. The contribution of the water species is not taken into account here. According to the spectrometric measurement the density fraction of water molecules was about 5\% (by density).

As it was expected, in this case the full energy species appeared to be significantly higher than that measured for the ion source with the RF plasma box. 


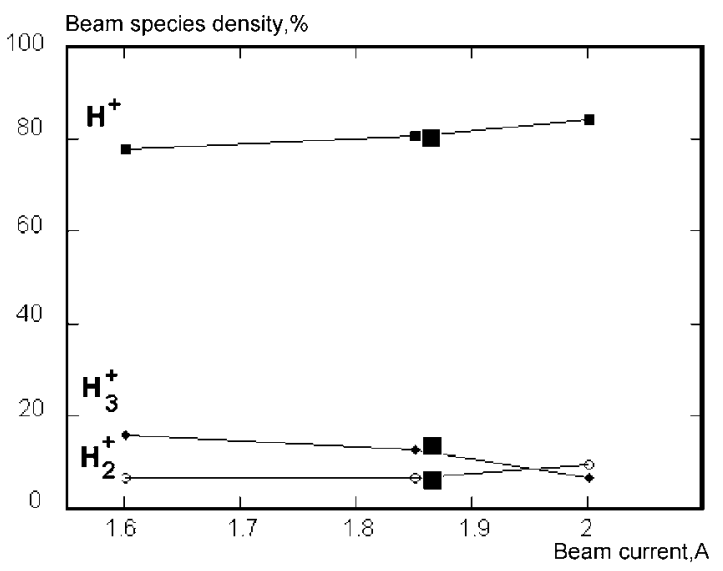

Figure 14. Ion species density vs extracted beam current.

\section{Conclusions}

The ion species mix was measured for the diagnostic hydrogen beam of the tokamak TEXTOR using the Doppler shift spectroscopy and, alternatively, by the magnetic mass spectrometer. Both methods provided reasonably consistent results. In the experiments, two types of the ion source were tested. The first was equipped with an RF plasma box. This ion source was identical to that operated at TEXTOR since 1998. The ion source routinely provides the $50 \mathrm{keV}, 1.8 \mathrm{~A}$ ion beam with $500 \mathrm{~Hz}$ modulation for $4 \mathrm{~s}$ (maximal pulse duration $10 \mathrm{~s}$ ). In the second version an arc-discharge plasma box was employed, which provided the same extracted current for maximally $2 \mathrm{~s}$, which was limited by the power loads onto plasma box components. It was found, that the arc-discharge based version provided a higher full energy fraction (> 75\%, by density) compared to the RF-based one ( $50 \%)$.

During the operation of the RF-version of the source certain degradation of the full energy species was observed. It can be attributed to a modification of the ceramic surface of the plasma box resulting in an enhanced recombination of atomic particles at the wall.

For the spectroscopic data interpretation we used the standard model [7] based on assumption of applicability of the Corona model, which assumes that collisional destruction of the excited states can be neglected and the collisional excitation is balanced by the radiative decay. Notwithstanding this model provided in our case reasonable agreement between the spectroscopic measurement data and those obtained with the magnetic mass spectrometer, further studies are necessary to evaluate the role of the effects of the collisional destruction, finite gas target 
thickness, axial non-uniformity of the gas in the observation region etc, which are not covered by the model. We plan to pursue these studies in the near future.

Acknowledgements. The authors are grateful to Dr.V.I.Davydenko from the Budker Institute for the helpful discussions. This work was supported in part by WTZ (Bilateral Scientific and Technological Collaboration) under research project \#RUS-567-99.

\section{References}

1. E.Hintz and B.Schweer, Plasma Phys. Controlled Fusion 37, 87 (1995).

2. M.Jakubovski, R.Fonk, J.S.Kim, and G.McKee. Rev. Sci. Instrum. 70, 874 (1998)

3. G.F.Abdrashitov, V.I.Davydenko, P.P.Deichul. D.J.Den Hartog, G.I.Fiksel, A.A.Ivanov, S.A.Korepanov, S.V.Murakhtin, and G.I.Shulzhenko. Rev. Sci. Instrum. 72, 594 (2001).

4. A.A.Ivanov, V.I.Davydenko, P.P.Deichui, A.Kreter, V.V.Mishagin, A.A.Podminogin, I.V.Shikhovtsev, B.Schweer, and R.Uhlemann. Rev. Sci. Instrum. 71, 3728 (2000).

5. C.F.Burrel, W.S.Cooper, R.R.Smith, and W.F.Steele. Rev. Sci. Instrum. 51, 1451 (1980).

6. J.F.Bonnal, G.Bracco, C.Breton, C.de Michelis, J.Druaux, M.Matioli, R.Oberson, and J.Ramette. J. Phys. D 15, 805 (1982).

7. R.Uhlemann, R.S.Hemsworth, G.Wang, and H.Euringer. Rev. Sci. Instrum. 64, 974 (1993).

8. A.Kreter and R.Uhlemann (1999) (not published).

9. S.J.Yoo, H.L.Yang, and S.M.Hwang. Rev. Sci. Instrum. 71, 1421 (2000).

10. B.J.Wood and H.Wise. J.Chem.Phys. 29, 1416 (1958), J. Chem.Phys. 65, 1976 (1961). 